\title{
An important landmarks construction for a GIS-Map based on indexing of dolly images
}

\author{
Abdulkadhem Abdulkareem Abdulkadhem, Tawfiq A. Al-Assadi \\ College of Information Technology, University of Babylon, Iraq
}

\begin{tabular}{|c|c|}
\hline Article Info & ABSTRACT \\
\hline Article history: & \multirow{10}{*}{$\begin{array}{l}\text { In this paper, we describe the construction of important landmarks of roads in } \\
\text { the GIS environment. The system uses the corners between more than two } \\
\text { roads as an important landmarks. In this corner points will be saving a } \\
\text { number of images, each one represents the movement direction between two } \\
\text { segment roads. The objective of our work is to build the geo-database } \\
\text { repository depend on the GIS (vector data) and multimedia (raster data) } \\
\text { information. This paper considered as a preprocessing step for a roadmap } \\
\text { discovery of video film when the input to the system is a dolly video film of } \\
\text { roads with absence the GPS locations of frames video, and the output is the } \\
\text { pathway (route) on the map inside the GIS environment. This work } \\
\text { considered as a starting point for multimedia query inside the GIS } \\
\text { environment. That's mean when query the GIS system about a particular } \\
\text { image or video, the GIS system must be able to know and determine the } \\
\text { location of this image or video file on the map. Thus, the first step for doing } \\
\text { this process needs to building and constructing an appropriate database for } \\
\text { matching process later. }\end{array}$} \\
\hline Received Dec 21, 2018 & \\
\hline Revised Jan 21, 2019 & \\
\hline Accepted Feb 28, 2019 & \\
\hline Keywords: & \\
\hline Geo-localization & \\
\hline GIS & \\
\hline Images & \\
\hline Maps & \\
\hline Video & \\
\hline
\end{tabular}

Copyright $\odot 2019$ Institute of Advanced Engineering and Science. All rights reserved.

\section{Corresponding Author:}

Abdulkadhem Abdulkareem Abdulkadhem,

College of Information Technology,

University of Babylon,

51002, Babil, Iraq.

Email:kazum2006k@yahoo.com

\section{INTRODUCTION}

GIS (Geographic Information System) are generally well-defined as an information system that analyzes, manipulates and manages the spatial data. GIS is used to produce information that is useful in decision making [1-6]. A GIS depends on an organized database that describes the world in geographic positions. A GIS is a unique kind of database of the world that represent a geographic database (geo-database), As part of a GIS geo-database design, users specify how certain features will be represented of ordered collections of vector-based features, Raster datasets, networks, terrains, and other surfaces and survey data sets. GIS datasets include traditional tabular attributes that define the geographic objects. These tabular information sets and relationships play a key role in GIS data models, just as they do in traditional database applications [7, 8].

The central to the GIS system is the database that is a collection of maps and associated information in digital form. Since the database is concerned with earth surface features, it can be seen to be involved of two components that are a spatial database describing the geography (shape and position) of earth surface features, and an attribute database describing the characteristics or qualities of these features. Thus, for example, we might have a property parcel defined in the spatial database and qualities such as its land use, owner, property valuation, and so on, in the attribute database [9, 10].

GIS fundamental component representations of spatial data are frequently well-defined in terms of points, lines, and areas. Nevertheless, additional kind of spatial data that is becoming commonly captured, 
but as yet is largely ignored in GIS, is that of video and images. While the digital video recording is a usually encountered medium in modern society and encompasses many forms, from the simple personal camera through to sophisticated survey and surveillance systems, its geographical representation in a GIS has not been fully examined or realized. In the majority of cases, the video film is commonly captured while the device and/or the objects being observed are in motion. What is of certain interest is when video streams can be, or have been, associated with spatial data such as location and orientation to generate geographically referenced video graphic data, which, for simplicity, will be defined as spatial video. Fundamentally, the nature of video is to record space, so when spatial properties can be accurately acquired and associated with this footage, an important geographical element can be considered for integration and analysis within a GIS $[11,12]$.

The media that represent spatial data may be extended to various media types such as aerial photo, graphics, attribute image, and satellite imagery. Such data types make users have richer information with various and complementary characteristics $[13,14]$. Modeling video in GIS has been largely ignored for numerous reasons such as data volumes involved in collecting and storing video footage. Thus, this paper suggests to indexing and storing small important video frames not exceeded $1 \%$ of the number of video frames in the GIS environment.

Recently, the most camcorders are mounted with GPS and enabled the linkage between video and GPS. Most videos present only graphics without a position of such video $[15,16]$. If the video can be visualized with its traveling path on GIS and snapshot of video can be gotten with its location, this will be very useful for GIS people to make use this media for updating GIS data [17, 18].

The basic motivation of this paper is how to build a database that can be used for determining the spatial location (geographic information) of a video or images imagine by a camcorder which is not mounted with GPS device in the GIS environment.

In the organization of the paper, Section 2, the proposed framework, which describes how the methodology presented Section 3, Experimental Results, gives an explanation of the output route highlighting and images displayed for important landmarks. In Section 4, Conclusions, where the authors reflect on the used method, achieved results and further improvements.

\section{THE PROPOSED FRAMEWORK} many steps.

The block diagram of the proposed framework for this paper is illustrated in Figure 1 that consists of

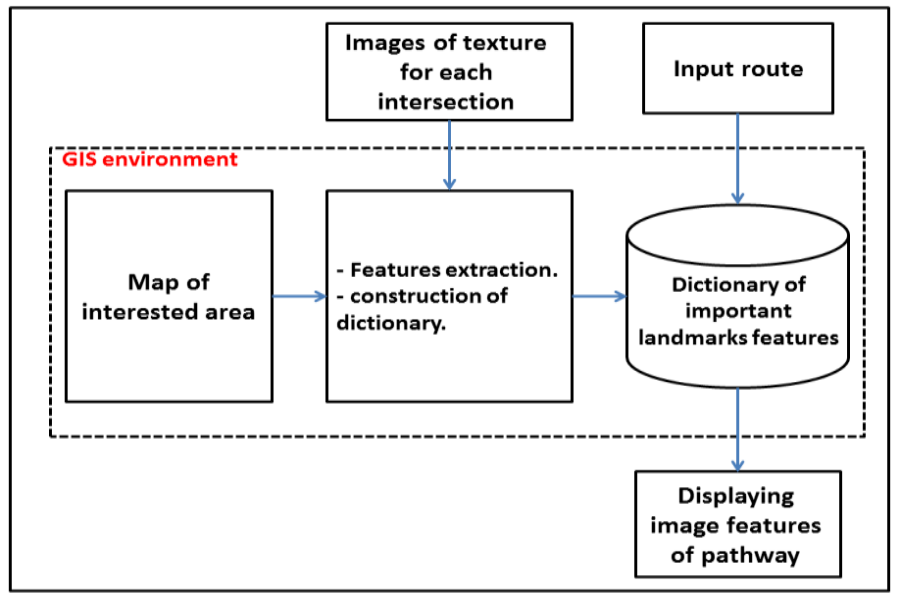

Figure 1. The block diagram of the proposed framework

\subsection{Map of Interested Area}

In the first step, we determine the workspace of the interested area inside the GIS environment. The information on the map (the interested area) are exported from the OpenStreetMap platform [19-21]. This platform can be given freely the information about (points that represent the important landmarks such as schools, hospital and government facilities, line that represent the roads, polygon that represents building) for any interested area in the world. The class features that used in our system is the points and lines. 
The data on the roadmap are extracted as a shape file of a polyline. From this shape file extracted their feature vertices and converted to its point features shape file. The feature points may need some rectification process by removing or adding some points feature. The output of this step is two shape files, the first one is a point feature that represent an intersection between at least two segment road (line), while the second one is the line features that represent the line between two intersection points.

\subsection{Images of Texture for Each Intersection}

In this step, will be taken a picture for each important landmark as a forward facing camera (the dolly forward movement [22-25]). The important landmark represents the intersection of at least two roads. The intersection can be represented as a point in the GIS environment. If the intersection point between two roads, that means we need to take two pictures for each side such as illustrated in Figure 2.

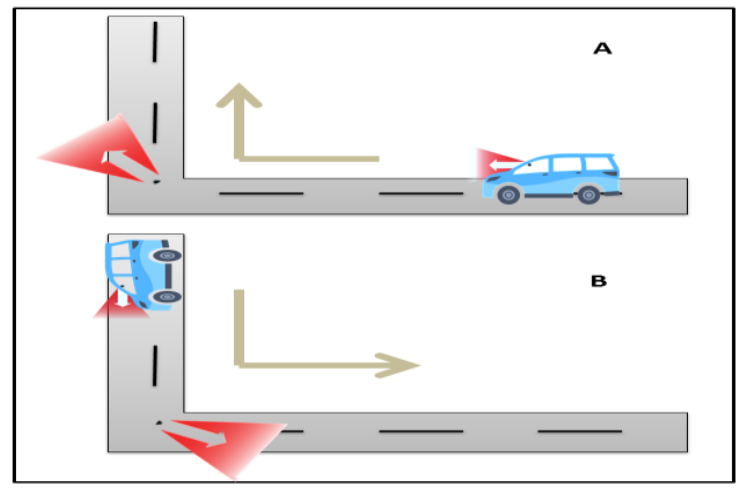

Figure 2. Taking a picture shot for an intersection between two roads

The picture is taken when there is a rotation to the left or to the right direction. We note in Figure 2-A the picture is taken for the texture environment at the moment that a car is moved from forward moving and change its direction to the right direction. In the Figure 2-B the picture is taken for the texture environment at the moment that a car is moved from forward moving and change its direction to the left direction. The common point (intersection or important landmark) in the above scenario has two pictures or images if the road has a two-way street, and one image if the road has one-way street. Now, what about if the important landmark represents the intersection between four roads. In this situation, the system needs to take (8) pictures for each direction change. Figure 3 explains this scenario.

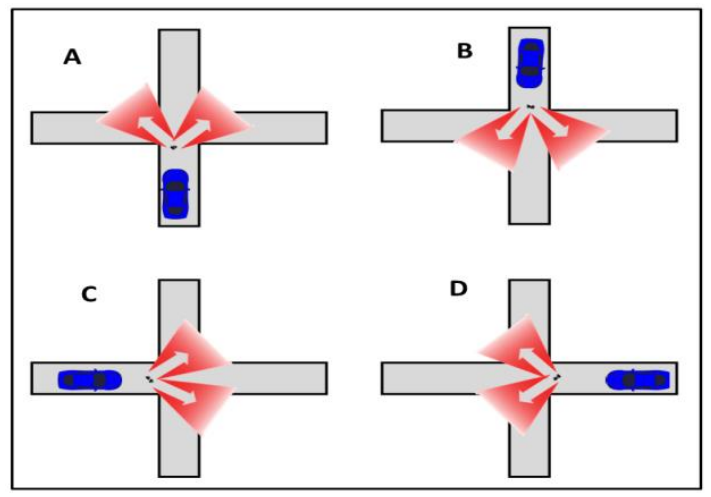

Figure 3. Taking pictures shot for an intersection between four roads

We notice in the Figure 3-A the car is moving from bottom to up. There are three possible moving of car before reach the intersection point, the first one is forward moving direction that does not need to take a picture shot, the second one is moving to the right direction which needs to take a picture shot, the last possible moving is to the left direction which also needs to take a picture shot. 


\subsection{Features Extraction and Construction of Dictionary}

In this step will be extracted the important landmarks on the roadmap in GIS and build the database table. There are two types of feature class are used for roads in the GIS environment. The first one is the point feature class that represents the intersection between more than two roads. The second one is the line feature class that represent road or segment between two intersection points. In our system, the road or street can be divided into the number of segments that represent the line between two intersections such as Figure 4.

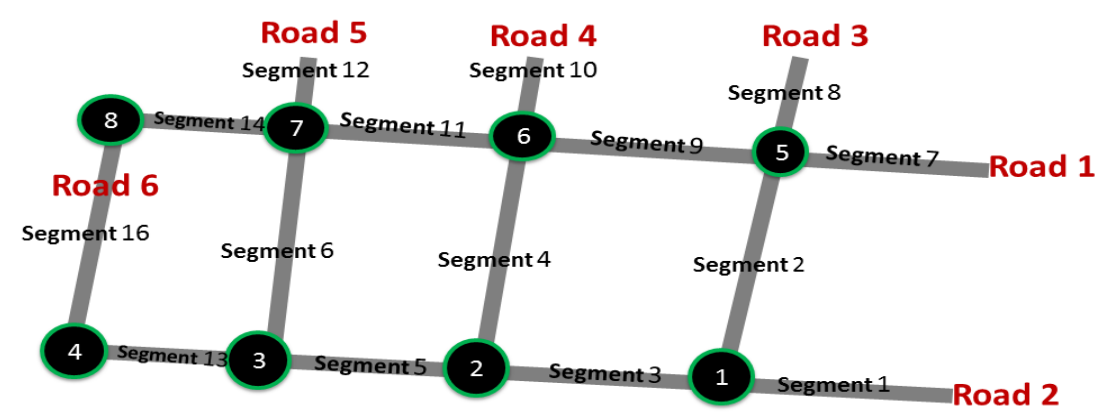

Figure 4. Example of roadmap representation

We notice from the Figure 4, there are six main roads (road 1, road 2, .., road 6). Each road has a number of intersection points that represent the important landmarks and also a number of segments. From the figure above, each of road (1) and road (2) has four intersection points and four segments. All of the road (3), road (4) and road (5) has two important landmarks and segments. The road (6) has two intersection points and one segment. The road (1) has four important landmarks that represent the intersection points $(5,6,7,8)$, and four segments $(7,9,11,14)$. The other roads can be interpreted in the same way. Each segment represents the line or road between two intersection points. Each intersection point represents the cross between more than two segments. If the intersection point between only two segments that means we need to save two images associated with this point feature class such as points (4 and 8) in the Figure 4. Each image represents one segment of road, in the case of point (4) the first image represents the segment (13) and the second image represent the segment (16). If the intersection point between three segments that means we need to save four images associated with this point feature class such as points (1, 2 and 3$)$. In the case of point (2), the first image represents segment (3), that means the picture is taken when moving from segment (4) to segment (3). The second image represents the segment (5), that means the picture is taken when moving from segment (4) to segment (5). The third and fourth images represent the segment (4), that means the third picture is taken when moving from segment (3) to segment (4) and the fourth picture is taken when moving from segment (5) to segment (4). If the intersection point between four segments that means we need to save eight images associated with this point feature class such as points $(5,6$ and 7$)$. In the case of point (5), each segment represented by two images such as Figure 3. Now we know how many numbers of images are needed to saving for each intersection points in the roadmap. The final operation of this step is to create a dictionary of important landmarks(intersection points) and each one has a number of segment connected to this intersection point and number of images that represent the neighbor segments. Table 1 represent the final output of this step as dictionary table (the database).

Table 1. The Dictionary Constriction of Figure (4)

\begin{tabular}{cccc}
\hline Important landmarks & Segment connected & No. of images & Images \\
\hline Point 1 & $1,2,3$ & 4 & $1,2,3,4$ \\
Point 2 & $3,4,5$ & 4 & $5,6,7,8$ \\
Point 3 & $5,6,13$ & 4 & $9,10,11,12$ \\
Point 4 & 13,16 & 2 & 13,14 \\
Point 5 & $7,8,9,12$ & 8 & $15,16,17,18,19,20,21,22$ \\
Point 6 & $4,9,10,11$ & 8 & $23,24,25,26,27,28,29,30$ \\
Point 7 & $6,11,12,14$ & 8 & $31,32,33,34,35,36,37,38$ \\
Point 8 & 14,16 & 2 & 39,40 \\
\hline
\end{tabular}




\subsection{The Input Route}

From the above steps, we extracted the important landmarks features from the roadmap of the interested area and taking a number of real pictures shot to these landmarks to build the database. The input to the system is a pathway depends on the segment number of the road rout. In Figure 5, there are three cases of the input rout step when the route (1) has the segments number $(1,2,9,4,5)$, while the route (2) has the segments number $(16,14,12)$ and route $(3)$ has the segments number $(8,9,4,3,2,7)$ consecutively.

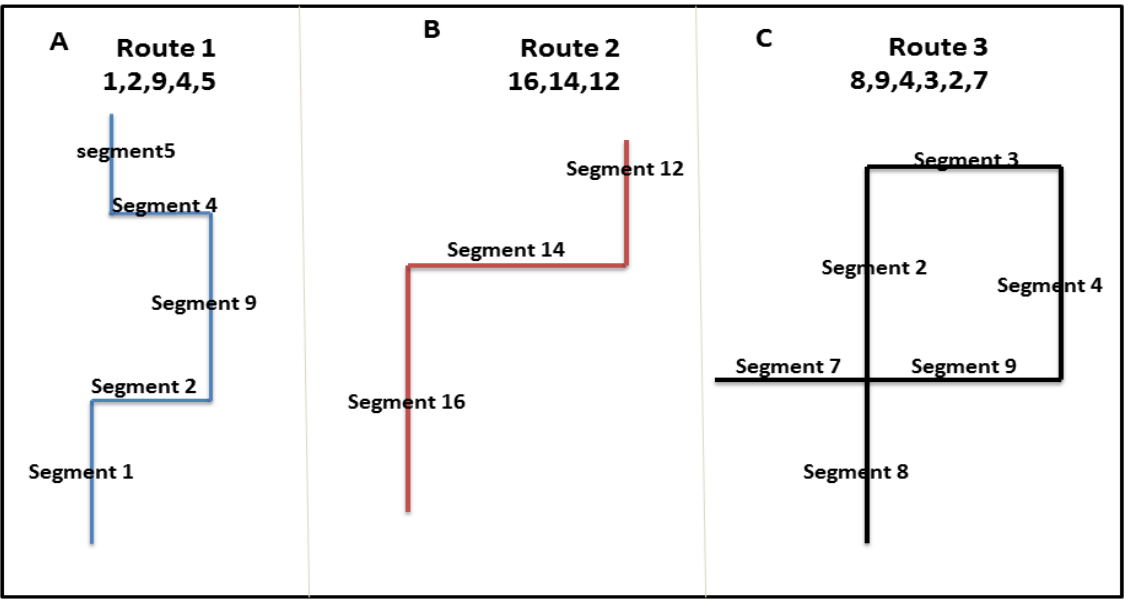

Figure 5. Example of input routes

\subsection{Displaying the Image Features of a Pathway}

In this step matching the segment road number of the above step with the constructed database for highlight the pathway and display the corresponding images when each image represents the movement from one segment to another. For example in the case (A) of the Figure 5, the input route is $(1,2,9,4,5)$. The first two segments are taken and extract the corresponding intersection point between them in the dictionary. We note the point (1) is the intersection between the sequential segment and it has four saving images (1-4) from the Table 1 . The image (1) represents the view between the segment $(1 \rightarrow 2)$, while the image (2) represents the view shot between the segment $(3 \rightarrow 2)$, images $(3,4)$ represent the view between the segments $(2 \rightarrow 1)$ and $(2 \rightarrow 3)$ consecutively. Thus, the image $(1)$ is displaced. The next two segments are $(2,9)$, thus the point between them segment is a point (5). We notice from the Table 1 there are eight images (15-22) saving in the dictionary each one represents the view or moving direction between two segments. These images have represented in the Table 2.

Table 2. The Images Representation of the Intersection Point (5)

\begin{tabular}{ccc}
\hline Images & From segment & To segment \\
\hline 15 & Segment 2 & Segment 7 \\
16 & Segment 2 & Segment 9 \\
17 & Segment 7 & Segment 2 \\
18 & Segment 7 & Segment 8 \\
19 & Segment 8 & Segment 7 \\
20 & Segment 8 & Segment 9 \\
21 & Segment 9 & Segment 2 \\
22 & Segment 9 & Segment 8 \\
\hline
\end{tabular}

From Table 2 we notice the image between (segment $2 \rightarrow$ segment 9) is an image (16). Next, take the next two consecutive segments $(9,4)$ to find the common point between them that is a point $(6)$. We notice from the Table 1 there are eight images (23-30) saving in this intersection point. These images are represented in a Table 3 . 
Table 3. The Images Representation of Intersection Point (6)

\begin{tabular}{ccc}
\hline Images & From segment & To segment \\
\hline 23 & Segment 4 & Segment 9 \\
24 & Segment 4 & Segment 11 \\
25 & Segment 9 & Segment 4 \\
26 & Segment 9 & Segment 10 \\
27 & Segment 10 & Segment 9 \\
28 & Segment 10 & Segment 11 \\
29 & Segment 11 & Segment 4 \\
30 & Segment 11 & Segment 10 \\
\hline
\end{tabular}

From Table 3 we notice the image between (segment $9 \rightarrow$ segment 4 ) is the image (25). Next, take the next two consecutive segments $(4,5)$ to find the common point between them that is a point (2). We notice from the Table 1 there are four images (5-8) saving in this intersection point. These images have represented in the Table 4.

Table 4. The Images Representation of the Intersection Point (2)

\begin{tabular}{ccc}
\hline Images & From segment & To segment \\
\hline 5 & Segment 3 & Segment 4 \\
6 & Segment 4 & Segment 3 \\
7 & Segment 4 & Segment 5 \\
8 & Segment 5 & Segment 4 \\
\hline
\end{tabular}

From Table 4 we notice the image between (segment $4 \rightarrow$ segment 5 ) is the image (7). Now, the output of the proposed framework is to highlight or flashing the segments input on the map and displaying list if images on that pathway or route. The list of images for the route of the case (A) on the Figure 5 that displayed on the map is (image 1, image 16, image 25 and image 7) such as illustrated in the Figure 6.

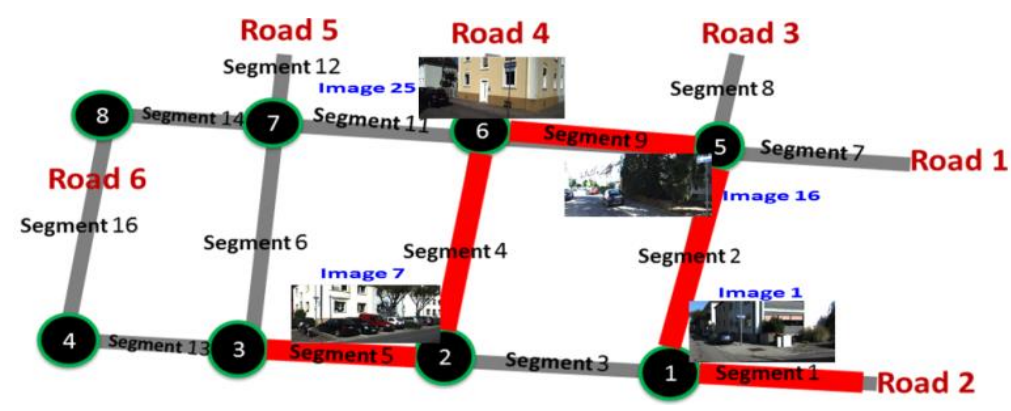

Figure 6 . The output display of the route $(1,2,9,4,5)$

For map display output of other cases routes in Figure 5 can be extracted as in the manner described above. Figure 7 and 8 illustrate the output of a map with images for the route $(16,14,12)$ and route $(8,9,4,3,2,7)$.

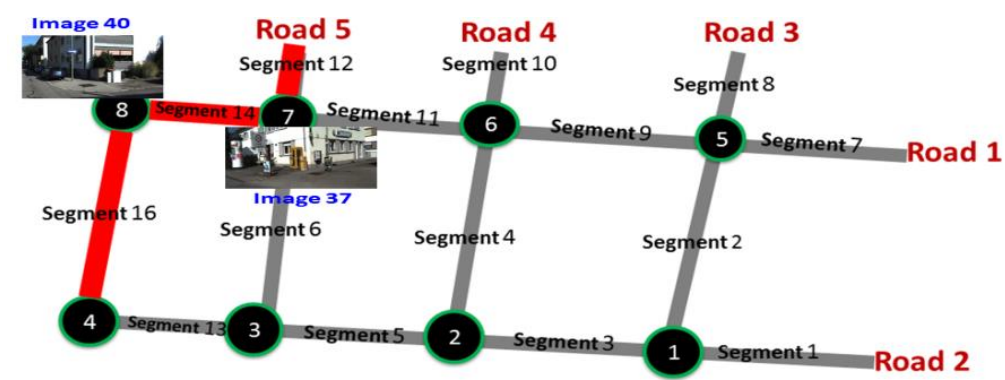

Figure 7. The output display of the route $(16,14,12)$ 


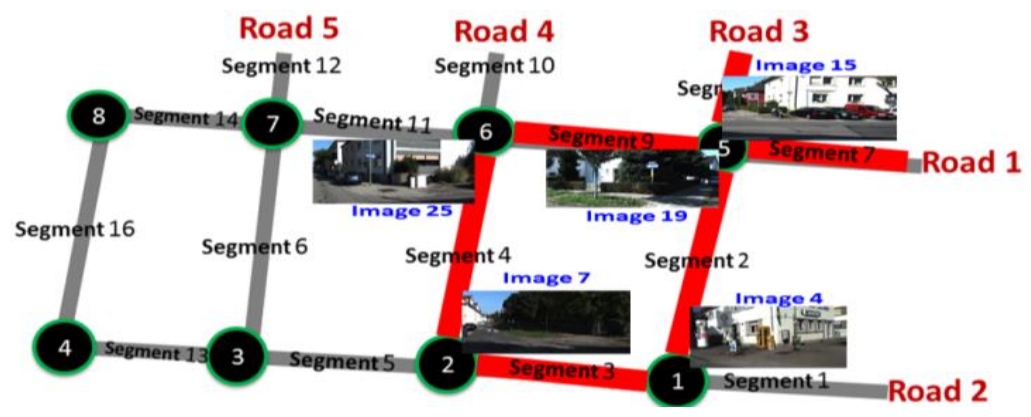

Figure 8 . The output display of the route $(8,9,4,3,2,7)$

\section{EXPERIENTIAL RESULTS}

Experiments of the proposed framework in this paper are performed on a small region of Karlsruhe city Baden-Württemberg-Germany. The information about roadmap is extracted from the OpenStreetMap platform as explains in Figure 9.

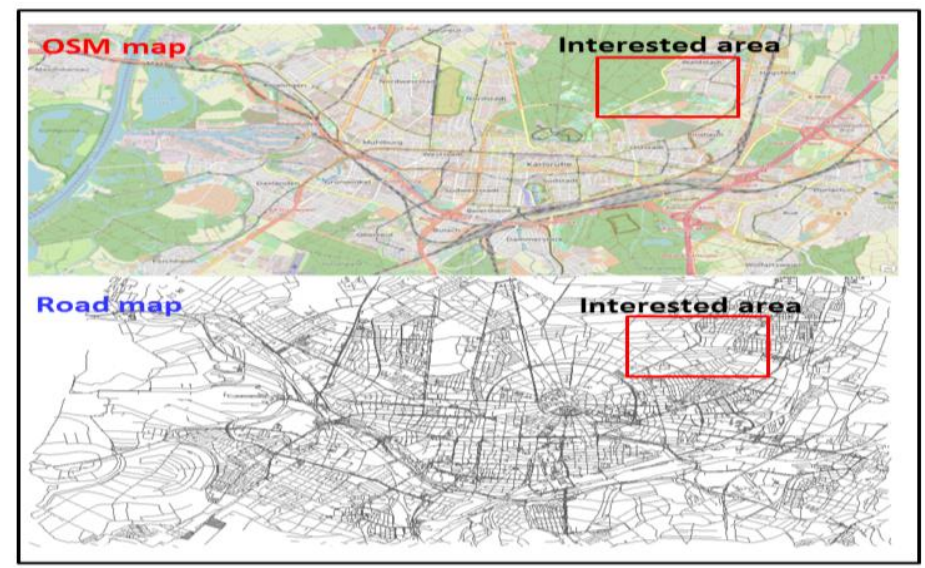

Figure 9. The interested area of Karlsruhe city from the OSM map.

There are many important landmarks (in our framework the important landmarks represent the intersection between roads). The intersection points are determined manually and take a number of picture shot for each point depends on the number of segment road connected to that point. Figure 10 represent the locations of the important landmarks on the map.

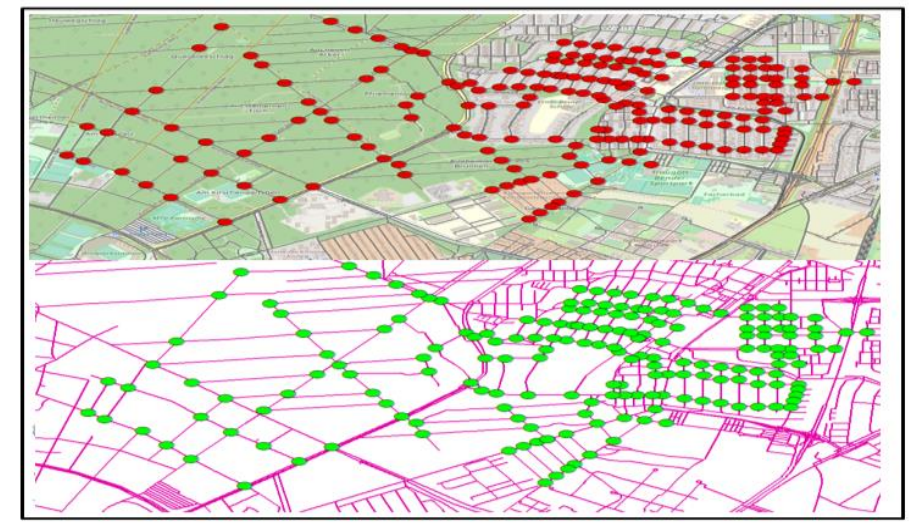

Figure 10. The important landmarks of the interested area. 
Pictures ware saved in these intersection points to build the dictionary database where each image has the location $(\mathrm{X}, \mathrm{Y})$ of their point affiliated to it. Each point may take (2, 4 or 8 ) images according to the segment road connected. There are (102) important landmarks point, (138) segment roads and (228) picture are saved for intersection points for the workspace area. Table 5 explains the dictionary of important landmarks.

Table 5. The Dictionary Constriction of the Interested Area in the Figure (10)

\begin{tabular}{ccc}
\hline Point & Segment road number & Images \\
\hline 1 & $1,2,3,4$ & $1,2,3,4,5,6,7,8$ \\
2 & $2,5,6$ & $9,10,11,12$ \\
3 & 3,7 & 13,14 \\
4 & $4,5,8,9$ & $15,16,17,18,19,20,21,22$ \\
$\cdot$ & $\cdot$ & $\cdot$ \\
$\cdot$ & $\cdot$ & $\cdot$ \\
102 & $136,137,138$ & $225,226,227,228$ \\
\hline
\end{tabular}

Now, to test our framework when the input route is $(71,69,67,64,62,59,58,56)$ segments. Figure 11 explain the final output that represents the highlighting the pathway route and important landmarks and also displays the images if you click on the intersection point.

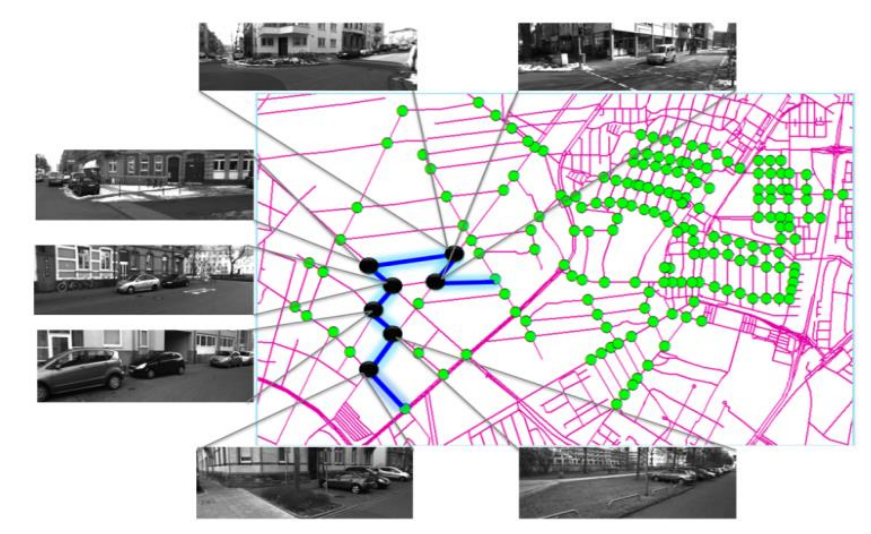

Figure 11. The output of the roadmap with important landmarks images

\section{CONCLUSIONS AND FUTURE WORKS}

An efficient fusion framework was effectively built and proved by combining two types of data environments, the first one is the GIS environment such as (maps, features class and etc.), while the second one is the multimedia environment such as (images and videos). The goal of this paper is how to build a database that can be used for determining the geographic information of a video. This work considered the starting point for multimedia query inside the GIS environment. That's mean when querying the GIS system about a particular image, the GIS system must know the location of this image on the map. In the future works, the construction database of this work can be used for extracting the locations on the GIS-map for road segments of the video film. The images that stored on the map dictionary may be used for the matching process with frames of video film based on appointed techniques.

\section{REFERENCES}

[1] Drew Decker. GIS Data sources. John Wiley \& Sons Inc, 2001.

[2] Abdelilah Azyat, Naoufal Raissouni, Nizar Ben Achhab, Mohamad Lahraoua and Asaad Chahboun. Mobile Geographic Information System Platform (MGISP): A GPS Information Collection System. International Journal of Information \& Network Security (IJINS). 2012; 1 (2): 140-151.

[3] Alias Abdul Rahman, Hairi Karim, Ivin A. Musliman, Bernad Siew, Hanis Rashidan,Sharif Idros, M. Al-Hafiz Azman, and M. Fadzli Abdullah. Developing GIS-Based Disaster Management System for Local Authority - Case Study in Malaysia. The International Archives of the Photogrammetry, Remote Sensing and Spatial Information Sciences, 2018; XLII-3(W4): 3-8. 
[4] Usman Ali, Mohammad Haris Shamsi, Cathal Hoare, James ODonnell. GIS-Based Residential Building Energy Modeling at District Scale. Proceedings of BSO 2018: 4th Building Simulation and Optimization Conference, Cambridge,2018:154-160.

[5] Hemlata Goyal, Chilka Sharma and Nisheeth Joshi. An Integrated Approach of GIS and Spatial Data Mining in Big Data. International Journal of Computer Applications, 2017; 169(11):1-6.

[6] Sayed Ahmed, Romani Farid Ibrahim and Hesham A. Hefny. GIS-Based Network Analysis for the Roads Network of the Greater Cairo Area. International Conference on Applied Research in Computer Science and Engineering ICAR'17, Lebanon, 2017.

[7] Michael Kennedy. Introducing Geographic Information Systems with ArcGIS. John Wiley \& Sons: New Jersey. 2006.

[8] J.M. MIRONGA. Geographic Information Systems (GIS) and Remote Sensing in the Management of Shallow Tropical Lakes. Applied Ecology and Environmental Research, 2004; 2(1): 83-103.

[9] J. Ronald Eastman. IDRISI Kilimanjaro Guide to GIS and Image Processing. Clark Labs. Clark University. USA, 2003.

[10] Mahmoudi Chokri, Mahmoudi Chokri, Flah Aymen and Sbita Lassaad. Smart database concept for power management in an electrical vehicle. International Journal of Power Electronics and Drive System (IJPEDS). 2019 ; 10 (1): 160-169.

[11] Paul Lewis, Stewart Fotheringham and Adam Winstanley. Spatial Video and GIS. National University of Ireland Maynooth. Maynooth. Co.Kildare. Ireland.

[12] Shunfu Hu. Multimedia GIS: Analysis and Visualization of Spatial and Temporal Geographic Information. International Archives of Photogrammetry and Remote Sensing, 2001; 34 (Part 4/W5): 42-47.

[13] Tae-Hyun Hwang, Kyoung-Ho Choi, In-Hak Joo and Jong-Hun Lee. MPEG-7 Metadata for Video-Based GIS Applications. IEEE. Spatial Information Technology Center. Korea. 2003.

[14] Toni Navarrete and Josep Blat. VideoGIS: Segmenting and indexing video based on geographic information, $5^{\text {th }}$ AGILE Conference on Geographic Information Science, Palma (Balearic Islands), Spain. 2002.

[15] In-Hak Joo, Tae-Hyun Hwang and Kyung-Ho Choi. Generation of video metadata supporting video - GIS integration. International conference on Image Processing (ICIP). Singapore. 2004.

[16] T. Someswari, Anil Kumar Tiwari, Nagaraj R. Novel Framework for Navigation using Enhanced Fuzzy Approach with Sliding Mode Controller. International Journal of Electrical and Computer Engineering (IJECE). 2018; 8 (6) : 4310-4320.

[17] Suchawadee Sillaparatl and Sanphet Chunithipaisan. CU-Video Tracker: QGIS Plugin For Video Tracker. Department of Survey Engineering. Chulalongorn University. Bangkok. Thailand. 2015.

[18] Keawseekao. Development of video image tool on Quantum GIS. The 18th National Convention on Civil Engineering (NCCE). Thailand. 2011.

[19] Website https://www.openstreetmap.org/, accessed October 2,2018.

[20] Zhiyong Wang and Lei Niu. A Data Model for Using OpenStreetMap to Integrate Indoor and Outdoor Route Planning. Sensors, 2018; 18(7): 1-19.

[21] Mordechai Haklay. How good is volunteered geographical information? Acomparative study of OpenStreetMap and Ordnance Survey datasets. Environment and Planning B: Planning and Design, 2010; 37 : 682-703.

[22] Website https://www.videomaker.com/article/c10/14221-camera-movement-techniques, accessed October 11,2018.

[23] H. Shishido, K. Yamanaka, Y. Kameda and I. Kitahara. Pseudo-Dolly-In Video Generation Combining 3D Modeling and Image Reconstruction. 2017 IEEE International Symposium on Mixed and Augmented Reality (ISMAR-Adjunct), 2017, Nantes: 327-333.

[24] Kyoko Katono and Michael Cohen. Dolly: zoom camera perspective with Alice. In Proceedings of the 13th International Conference on Humans and Computers (HC '10), University of Aizu Press, Fukushima-ken, Japan, 55-58.

[25] V K Siswanto, B U Aulia, E B Santoso, E Umilia and N Zakina. Potential and problems participatory mapping of creative industry in Kampong Dolly, Surabaya. IOP Conf. Series: Earth and Environmental Science,2018; 1-8. 